OPEN ACCESS

Edited by: Lorenzo Manti, University of Naples Federico II, Italy

Reviewed by: Valentina Lancellotta, Catholic University of the Sacred Heart, Italy

Uzoma Kevin Iheagwara, University of Pittsburgh Medical

Center, United States

*Correspondence: Xishan Wang wxshan1208@126.com Xu Guan drguanxu@126.com

Specialty section: This article was submitted to

Radiation Oncology,

a section of the journal

Frontiers in Oncology

Received: 16 July 2020 Accepted: 05 October 2020 Published: 29 October 2020

Citation: Yang R, Guan X, Liu E, Wei R, Zhao Z, Chen $H$, Liu Z, Yang $M$, Jiang $Z$ and Wang $X$ (2020) Risk and Prognosis of Secondary Rectal Cancer After Radiation Therapy for Pelvic Cancer.

Front. Oncol. 10:584072. doi: 10.3389/fonc.2020.584072

\section{Risk and Prognosis of Secondary Rectal Cancer After Radiation Therapy for Pelvic Cancer}

\author{
Runkun Yang ${ }^{1}$, Xu Guan ${ }^{2 *}$, Enrui Liu ${ }^{2}$, Ran Wei ${ }^{2}$, Zhixun Zhao ${ }^{2}$, Haipeng Chen ${ }^{2}$, \\ Zheng Liu ${ }^{2}$, Ming Yang ${ }^{2}$, Zheng Jiang ${ }^{2}$ and Xishan Wang ${ }^{1,2,3^{*}}$ \\ ${ }^{1}$ Department of Colorectal Surgery, The Second Affiliated Hospital of Harbin Medical University, Harbin, China, ${ }^{2}$ Department \\ of Colorectal Surgery, National Cancer Center/Cancer Hospital/National Clinical Research Center for Cancer, Chinese \\ Academy of Medical Sciences and Peking Union Medical College, Beijing, China, ${ }^{3}$ Colorectal Cancer Institute, Harbin \\ Medical University, Harbin, China
}

Background: The relationship between pelvic radiation therapy (RT) and second primary rectal cancer (SPRC) is unclear. The aim of this study was to assess the risk and prognosis of SPRC after pelvic RT.

Materials and Methods: Data for patients who had primary pelvic cancer (PPC) between 1973 and 2016 were retrieved from the Surveillance, Epidemiology, and End Results (SEER) database. Multiple primary standardized incidence ratios (SIRs) were used to assess the risk of SPRC. Five-year overall survival (OS) and rectal cancer-specific survival (RCSS) were calculated using Kaplan-Meier curves.

Results: A total of 573,306 PPC patients were included, 141,225 of whom had been treated with RT. Primary cancers were located in the prostate $(50.83 \%)$, bladder (24.18\%), corpus uterus (16.26\%), cervix (5.83\%), and ovary $(2.91 \%)$. A total of 1,491 patients developed SPRC. Overall, the patients who received RT were at increased risk of developing SPRC (SIR = 1.39, 95\% confidence interval [Cl]: 1.27-1.52). The risk of SPRC decreased in patients who did not undergo RT (SIR $=0.85,95 \% \mathrm{Cl}$ : 0.80-0.91). The SIR for SPRC in patients who underwent external beam radiation therapy (EBRT) was 1.22 (95\% Cl: 1.09-1.36). The SIR for SPRC in patients who underwent a combination of EBRT and brachytherapy (EBRT-BRT) was 1.85 (95\% Cl: 1.60-2.14). For patients who received $\mathrm{RT}$, the SIR for SPRC increased with time after a 5-year latency period from PPC diagnosis. The survival of RT-treated SPRC patients was significantly worse than that of patients with primary rectal cancer only (PRCO).

Conclusions: Patients receiving pelvic RT were at an increased risk of developing SPRC. Different pelvic RT treatment modalities had different effects on the risk of SPRC. We suggest that long-term surveillance of SPRC risk is required for patients who have undergone pelvic RT, especially young patients.

Keywords: pelvic cancer, radiation therapy, second primary cancer, rectal cancer, Surveillance; Epidemiology; End Results database 


\section{INTRODUCTION}

Radiation therapy (RT) has formed part of the treatment regimen for at least $50 \%$ of all cancer patients because of the associated reduction in recurrence and improved prognosis (1, 2). RT destroys cancer cells by depositing high-energy radiation on cancer tissues. High doses of ionizing radiation can directly or indirectly (through the production of free radicals) damage the genome of the cell (3). Nevertheless, acute and late toxicity due to RT cannot be ignored.

The risk of developing a second primary cancer (SPC) is one form of late toxicity (4). Nearly 1 in 12 patients diagnosed with a common cancer develop a SPC. More than 55\% of patients with two incident cancers die as a result of a secondary cancer (5). During RT for pelvic cancer, the rectum is likely to receive more radiation than organs in the non-pelvic area (6-8), and it is important to understand how radiation affects SPC risk within the field of irradiation.

Studies have shown conflicting results for second primary rectal cancer (SPRC) risk after pelvic RT (9-11). A study based on a Dutch population demonstrated that patients receiving pelvic RT were at an increased risk of developing a SPRC (12), while other studies reported that the tumor incidence in patients receiving pelvic RT did not differ from that of the general population $(13,14)$. These observations indicate that the relationship between pelvic RT and SPRC requires further determination. Theoretically, different RT modalities may have different effects on SPRC risk; however, there is currently insufficient evidence to reach such a conclusion (15-17).

Compared with primary rectal cancer (PRC), the etiology of RT-related SPRC can be very different. Moreover, whether SPRCs and PRCs are equally sensitive to RT after pelvic RT treatment remains unclear (18). In addition, fibrosis resulting from pelvic RT might make complicate surgery and lead to an increase in surgery-related complications (19). Because of these differences, the survival outcomes between PRC and RT-related SPRC patients might be different.

The aims of the present study were to identify how pelvic RT affects the risk of developing SPRC and compare the survival outcome between PRC and RT-related SPRC patients.

\section{METHODS}

\section{Data Source}

The processed, publicly available data on the SEER database was access between January 1, 1973, and December 31, 2016, from 9 registries (Atlanta, Connecticut, Detroit, Hawaii, Iowa, New Mexico, San Francisco-Oakland, Seattle-Puget Sound, and Utah). The demographic and incidence data collected by the SEER registries cover approximately $28 \%$ of the US population, and are considered representative of the US population as a whole. Because patients' records in the SEER database were anonymized and de-identified before analysis, information on cancer cases can be retrieved from the database. The study design was approved by the Ethics Committee of the National Cancer Center/National Clinical Research Center for Cancer/Cancer
Hospital; the Chinese Academy of Medical Sciences and Peking Union Medical College institutional review board; and the Second Affiliated Hospital of Harbin Medical University review board. All the methods performed in our study followed the latest guidelines stated in the SEER database.

\section{Identification of First Primary Pelvic Cancer (PPC)}

Solid pelvic cancers in five sites that are routinely treated with RT were included as first primary cancers in this cohort study, including cancers of the cervix uteri, corpus and uterus, ovary, prostate, and urinary bladder. The SEER database strictly adheres to the coding rules for the classification of topography or histology of the International Classification of Diseases for Oncology (ICD-O) guidelines to identify multiple primary malignancies and distinguish between primary and recurrent cancers. The exclusion criteria for a first pelvic primary cancer in this study were as follows: patients with distant cancers; patients under 20 years of age; and patients of unknown race, survival month, cause of death, or RT status.

\section{Treatment for PPC}

The SEER database contains information on the first course of treatment. In this study, patients with a first PPC were classified according to the type of RT received, including external beam radiation therapy (EBRT), a combination of EBRT with brachytherapy involving implants or isotopes (EBRT-BRT), and no radiation therapy (NRT). However, the dosages of radiation administered were not registered in the SEER database.

\section{Identification of SPRC}

Because it takes 5 years from radiation exposure to solid cancer induction (20), the primary outcome of interest was the development of SPRC or second primary rectosigmoid cancer, which was defined as a nonsynchronous malignancy occurring within 5 years after treatment of the first PPC. In addition, patients diagnosed with a third-order or higher multiple PRC were excluded from the study.

\section{Identification of Primary Rectal Cancer Only (PRCO)}

Patients with PRCO were defined as those presenting only with PRC, and without any other malignancy diagnosed during their lifetime.

\section{Statistical Analysis}

Baseline patient and tumor characteristics were compared using the $\chi^{2}$ test or Fisher's exact test, in case of an expected cell count $<5$. Survival outcomes were calculated using Kaplan-Meier curves, and the log-rank test was applied to compare these curves. The definition of overall survival (OS) was the time from SPRC diagnosis to the date of all-cause death, and the definition of rectal cancer-specific survival (RCSS) was the time from SPRC diagnosis to the date of SPRC-cause death.

SPSS (version 22.0; IBM) was used for the analysis of characteristics and survival, and a $p$-value $<0.05$ was considered statistically significant. Multiple primary standardized incidence 
ratios (SIRs) were used as a key measure of the risk of developing a SPC. Here, SIR was defined as the ratio of SPRC incidence to the number of expected SPRC cases in the general US population according to the SEER ascertainment area. The SIR results were stratified by gender, age, and calendar time, and a $p$-value $<0.05$ (two-sided) was considered statistically significant. Exact Poisson methods were used to calculate $95 \%$ confidence intervals (CIs) for the ratio of observed events to expected events (21). All SIR analyses were conducted using SEER ${ }^{\star}$ Stat software, version 8.3.6.d.

\section{RESULTS}

\section{Patient Characteristics}

We identified 573,306 patients meeting the selection criteria (average age, 65 years). Patient demographics are depicted in Table 1. A total of 141,225 patients $(24.63 \%)$ were treated with RT for a PPC, 103,947 of which were treated with EBRT only, and 37,278 with EBRT-BRT. Meanwhile, 432,081 (75.37\%) patients received no RT. Primary cancers were located in the prostate $(50.83 \%)$, bladder $(24.18 \%)$, corpus uteri $(16.26 \%)$, cervix $(5.83 \%)$, and ovary $(2.91 \%)$.

After a minimum latency of five years from PPC diagnosis, a total of 91,359 patients (15.94\%) developed a SPC. Among patients who underwent RT, $15.65 \%$ went on to develop a SPC. In total, 16,366 (15.74\%) patients in the EBRT group and $5,740(15.40 \%)$ patients in the EBRT-BRT group developed a SPC. In the NRT group, 69,253 (16.03\%) patients developed a SPC. The number of patients who developed a SPRC was 1,491 $(0.23 \%), 325(0.31 \%)$, and $187(0.50 \%)$ for the NRT, EBRT, and EBRT-BRT groups, respectively. The above data showed that, compared with the NRT group, a greater proportion of patients who received EBRT and EBRT-BRT for their PPC developed a SPRC; no difference was found between the latter two groups.

\section{Comparison of SPRC With and Without RT}

The characteristics of the patients who developed a SPRC after a minimum latency of five years from PPC diagnosis are shown in Table 2. Patients who developed a SPRC after RT (RT-SPRC) were older than those who developed a SPRC without RT (NRTSPRC) $(76.20 \pm 9.20 v s .74 .74 \pm 10.29, p=0.007)$. There was no significant difference between the NRT-SPRC group and the RTSPRC group in terms of gender, race, tumor grade, and whether the patients underwent surgery or not. The proportion of SPRCs that were located in the rectum in the RT-SPRC group was significantly higher than that in the NRT-SPRC group (81.25 vs. $72.93 \%, p<0.001)$. Patients in the RT- SPRC group had a greater proportion of mucinous adenocarcinomas $(8.59 \%)$ than those in the NRT-SPRC group $(4.80 \%)(p=0.001)$. Compared with those in the NRT-SPRC group, patients in the RT-SPRC group had a smaller proportion of localized stages (50.75 vs. $48.13 \%$, respectively; $p=0.045$ ) and a greater proportion of regional stages ( 33.41 vs. $36.68 \%$, respectively; $p=0.045$ ). A significantly greater percentage of patients in the NRT-SPRC group received chemotherapy for their SPRC compared with those from the RTSPRC group (32.89 vs. $26.17 \%$, respectively; $p=0.009$ ). Only
9.75\% of the patients in the RT-SPRC group received RT again for their SPRC. However, $30.34 \%$ of the patients in the NRTSPRC group received RT for their SPRC.

\section{SIR of SPRC}

Compared with the general US population, patients who received RT for their PPC were at an increased risk of developing a SPRC $(\mathrm{SIR}=1.39,95 \%$ CI: $1.27-1.52)$. The SIR of SPRC was $1.22(95 \%$ CI: 1.09-1.36) in the EBRT group and 1.85 (95\% CI: 1.60-2.14) in the EBRT-BRT group (Table 3). The increased risk of developing a SPRC was due to RT, as evidenced by the SIR of 1.39 in the RT group compared with a SIR of 0.85 for the NRT group (95\% CI: $0.80-0.91$ ). The SIR of SPRC tended to be higher in patients who underwent EBRT and EBRT-BRT than in patients who had not received RT. This tendency was found for the cervix uteri, corpus and uterus, prostate, and bladder, but not the ovary. Among patients who did not receive RT, those with prostate cancer (SIR $=0.75,95 \% \mathrm{CI}: 0.67-0.8$ ) and bladder cancer (SIR $=0.84,95 \%$ CI: $0.75-0.94)$ were at a reduced risk of developing a SPRC.

We next calculated the SIRs of SPRC according to age range, gender, race, latency from PPC diagnosis, year of diagnosis, and PPC site, respectively. NRT and EBRT-BRT patients presented a tendency for a deceasing risk of developing SPRC with increasing age at diagnosis (Figure 1A). This showed that patients who were younger at PPC diagnosis were at an increased risk of developing SPRC. Furthermore, we found that the risk of developing SPRC increased with time after a 5-year latency from the diagnosis of PPC in the EBRT and EBRT-BRT groups, but not in the NRT group (Figure 1B). Additionally, we found that the risk of developing a SPRC increased with increasing calendar year of diagnosis (Figure 1C).

\section{Survival Outcomes for SPRC Patients}

We separately matched NRT-SPRC and RT-SPRC patients with PRCO patients by age at diagnosis, gender, race, tumor stage, chemotherapy, radiotherapy, and surgery by propensity score matching (PSM) at a ratio of 1:5. Demographic data of patients after PSM were shown in Supplementary Data.

Significant differences in five-year OS were found between NRT-SPRC patients and matched PRCO patients $(p=0.002$; Figure 2A). We also found that the 5-year OS for RT-SPRC patients was significantly shorter than that for matched PRCO patients $(p<0.001$; Figure 2B). The hazard ratio (HR) was 1.18 (95\% CI: 1.06-1.31) for the NRT-SPRC group vs. the matched PRCO group and 1.33 (95\% CI: 1.14-1.55) for the RT-SPRC vs. the matched PRCO group. In addition, the survival analysis showed that there was a significant difference in RCSS between RT-SPRC patients and matched PRCO patients $(p=0.004, \mathrm{HR}=$ 1.30, 95\% CI: 1.07-1.58; Figure 2D). No significant difference in RCSS was observed between patients in the NRT-SPRC group and those in the PRCO group $(p=0.116 ; \mathrm{HR}=1.11,95 \%$ CI: 0.97-1.28; Figure 2C). These results suggested that the prognosis was worse for RT-receiving SPRC patients than for PRCO patients. 
TABLE 1 | Characteristics of the primary pelvic cancer (PPC) patients.

\begin{tabular}{|c|c|c|c|c|c|c|c|c|c|c|}
\hline & \multirow{2}{*}{\multicolumn{2}{|c|}{$\begin{array}{c}\text { Total } \\
N=574,253\end{array}$}} & \multirow{2}{*}{\multicolumn{2}{|c|}{$\begin{array}{c}\text { NRT } \\
N=432,422\end{array}$}} & \multirow{2}{*}{\multicolumn{2}{|c|}{$\begin{array}{c}\text { RT } \\
N=141,831\end{array}$}} & \multicolumn{4}{|c|}{ RT Type } \\
\hline & & & & & & & & $\begin{array}{c}\text { EBRT } \\
n=104,194\end{array}$ & & $\begin{array}{l}\text { EBRT-BRT } \\
n=37,637\end{array}$ \\
\hline & $n^{\mathrm{a}}$ & $n(\%)$ or mean \pm SD & $n^{\mathrm{a}}$ & $n(\%)$ or mean \pm SD & $n^{\mathrm{a}}$ & $n(\%)$ or mean \pm SD & $n^{\mathrm{a}}$ & $n(\%)$ or mean \pm SD & $n^{\mathrm{a}}$ & $n(\%)$ or mean \pm SD \\
\hline Age at diagnosis & 573,306 & $\begin{array}{c}65.00 \\
\pm \\
12.36\end{array}$ & 432,081 & $\begin{array}{c}64.67 \\
\pm \\
12.75\end{array}$ & 141,225 & $\begin{array}{c}66.02 \\
\pm \\
11.04\end{array}$ & 103,947 & $\begin{array}{c}66.02 \\
\pm 11.04\end{array}$ & 37,278 & $\begin{array}{c}62.02 \\
\pm \\
12.11\end{array}$ \\
\hline$\leq 60$ & & 191,026 (33.32) & & $152,976(35.40)$ & & $38,050(26.94)$ & & 23,165 (22.29) & & 14,885 (39.93) \\
\hline$>60$ & & $382,280(66.68)$ & & $279,105(64.60)$ & & $103,175(73.06)$ & & $80,782(77.71)$ & & $22,393(60.07)$ \\
\hline Gender & 573,306 & & 432,081 & & 141,225 & & 103,947 & & 37,278 & \\
\hline Male & & 395,647 (69.01) & & $297,433(68.84)$ & & $98,214(69.54)$ & & $79,563(76.54)$ & & $18,651(50.03)$ \\
\hline Female & & 177,659 (30.99) & & $134,648(31.16)$ & & $43,011(30.46)$ & & 24,384 (23.46) & & $18,627(49.97)$ \\
\hline Race & 573,306 & & 432,081 & & 141,225 & & 103,947 & & 37,278 & \\
\hline White & & 479,744 (83.68) & & 369,500 (85.52) & & 110,244 (78.06) & & 81,755 (78.65) & & 28,489 (76.42) \\
\hline Black & & $57,981(10.11)$ & & $37,817(8.75)$ & & $20,164(14.28)$ & & 14,296 (13.75) & & $5,868(15.74)$ \\
\hline Other & & $35,581(6.21)$ & & 24,764 (5.73) & & $10,817(7.66)$ & & $7,896(7.60)$ & & $2,921(7.84)$ \\
\hline SPC & 573,306 & & 432,081 & & 141,225 & & 103,947 & & 37,278 & \\
\hline Yes & & 91,359 (15.94) & & $69,253(16.03)$ & & 22,106 (15.65) & & 16,366 (15.74) & & $5,740(15.40)$ \\
\hline No & & 481,947 (84.06) & & $362,828(83.97)$ & & $119,119(84.35)$ & & 87,581 (84.26) & & 31,538 (84.60) \\
\hline SPRC & 573,306 & & 432,081 & & 141,225 & & 103,947 & & 37,278 & \\
\hline Yes & & 1,491 (0.26) & & $979(0.23)$ & & $512(0.36)$ & & $325(0.31)$ & & $187(0.50)$ \\
\hline No & & 571,815 (99.74) & & $431,102(99.77)$ & & $140,713(99.64)$ & & 103,622 (99.69) & & 37,091 (99.50) \\
\hline Site & 573,306 & & 432,081 & & 141,225 & & 103,947 & & 37,278 & \\
\hline Cervix & & $33,428(5.83)$ & & 17,003 (3.94) & & $16,425(11.63)$ & & $5,930(5.70)$ & & $10,495(28.15)$ \\
\hline Corpus uteri & & 93,201 (16.26) & & 69,980 (16.20) & & $23,221(16.44)$ & & $15,128(14.55)$ & & $8,093(21.71)$ \\
\hline Ovary & & $16,680(2.91)$ & & $15,861(3.67)$ & & $819(0.58)$ & & $795(0.76)$ & & $24(0.06)$ \\
\hline Prostate & & $291,395(50.83)$ & & $199,655(46.21)$ & & $91,740(64.96)$ & & 73,106 (70.33) & & $18,634(49.99)$ \\
\hline Bladder & & $138,602(24.18)$ & & $129,582(29.99)$ & & $9,020(6.39)$ & & $8,988(8.65)$ & & $32(0.09)$ \\
\hline Grade & 516,100 & & 386,043 & & 130,057 & & 97,798 & & 32,259 & \\
\hline$|\sim| \mid$ & & 315,974 (61.22) & & 249,194 (64.55) & & $66,780(51.35)$ & & $49,537(50.65)$ & & $17,243(53.45)$ \\
\hline III IV & & $200,126(38.78)$ & & $136,849(35.45)$ & & 63,277 (48.65) & & 48,261 (49.35) & & $15,016(46.55)$ \\
\hline Stage & 573,306 & & 432,081 & & 141,225 & & 103,947 & & 37,278 & \\
\hline Localized & & 221,450 (38.63) & & 198,864 (46.02) & & 22,586 (15.99) & & 14,717 (14.16) & & 7,869 (21.11) \\
\hline Regional & & $60,461(10.55)$ & & $33,562(7.77)$ & & $26,899(19.05)$ & & $16,124(15.51)$ & & $10,775(28.90)$ \\
\hline Localized/regional (prostate-related cases) & & $291,395(50.83)$ & & $199,655(46.21)$ & & $91,740(64.96)$ & & $73,106(70.33)$ & & $18,634(49.99)$ \\
\hline Chemotherapy & 573,306 & & 432,081 & & 141,225 & & 103,947 & & 37,278 & \\
\hline Yes & & $40,210(7.01)$ & & $27,807(6.44)$ & & $12,403(8.78)$ & & $8,000(7.70)$ & & 4,403 (11.81) \\
\hline No & & $533,096(92.99)$ & & $404,274(93.56)$ & & $128,822(91.22)$ & & $95,947(92.30)$ & & $32,875(88.19)$ \\
\hline Surgery & 569,351 & & 429,758 & & 139,593 & & 103,154 & & 36,439 & \\
\hline Yes & & $393,432(69.10)$ & & $346,820(80.70)$ & & 46,612 (33.39) & & 35,393 (34.31) & & 11,219 (30.79) \\
\hline No & & $175,919(30.90)$ & & 82,938 (19.30) & & $92,981(66.61)$ & & $67,761(65.69)$ & & $25,220(69.21)$ \\
\hline
\end{tabular}

a Number of patients for whom data was available.

NRT, no radiation therapy; RT, radiation therapy; EBRT, external beam radiation therapy; EBRT-BRT, combination of external beam with brachytherapy; SPC, second primary cancer; SPRC, second primary rectal cancer. 
TABLE 2 | Comparison of the characteristics of second primary rectal cancer (SPRC) patients receiving radiotherapy (RT) or not.

\begin{tabular}{|c|c|c|c|c|c|}
\hline & \multicolumn{2}{|c|}{$\begin{array}{c}\text { NRT-SPRC } \\
n=979\end{array}$} & \multicolumn{2}{|c|}{$\begin{array}{c}\text { RT-SPRC } \\
n=512\end{array}$} & \multirow[t]{2}{*}{$p$-value } \\
\hline & $n^{\mathrm{a}}$ & $n(\%)$ or mean \pm SD & $n^{\mathrm{a}}$ & $n(\%)$ or mean \pm SD & \\
\hline Age at diagnosis & 979 & $74.74 \pm 10.29$ & 512 & $76.20 \pm 9.20$ & 0.007 \\
\hline$\leq 60$ & & $96(9.81)$ & & $30(5.86)$ & \\
\hline$>60$ & & 883 (90.19) & & $482(94.14)$ & \\
\hline Gender & 979 & & 512 & & 0.836 \\
\hline Male & & $632(64.56)$ & & $327(63.87)$ & \\
\hline Female & & $347(35.44)$ & & $185(36.13)$ & \\
\hline Race & 979 & & 512 & & 0.178 \\
\hline White & & $837(85.50)$ & & 419 (81.84) & \\
\hline Black & & $74(7.56)$ & & $50(9.77)$ & \\
\hline Other & & $68(6.95)$ & & $43(8.40)$ & \\
\hline Site & 979 & & 512 & & $<0.001$ \\
\hline Rectum & & $714(72.93)$ & & $416(81.25)$ & \\
\hline Rectosigmoid junction & & $265(27.07)$ & & $96(18.75)$ & \\
\hline Pathological type & 979 & & 512 & & 0.001 \\
\hline Adenocarcinoma & & $831(84.88)$ & & $436(85.16)$ & \\
\hline Mucinous adenocarcinoma & & $47(4.80)$ & & 44 (8.59) & \\
\hline Other & & $101(10.32)$ & & $32(6.25)$ & \\
\hline Grade & 775 & & 413 & & 0.43 \\
\hline $\mid \sim \|$ & & $632(81.55)$ & & $326(78.93)$ & \\
\hline III IV & & $143(18.45)$ & & $87(21.07)$ & \\
\hline Stage & 865 & & 428 & & 0.045 \\
\hline Localized & & $439(50.75)$ & & $206(48.13)$ & \\
\hline Regional & & 289 (33.41) & & 157 (36.68) & \\
\hline Distant & & $137(15.84)$ & & 65 (15.19) & \\
\hline Tumor size & 394 & & 239 & & 0.017 \\
\hline$\leq 2 \mathrm{~cm}$ & & 89 (22.59) & & 39 (16.32) & \\
\hline$>2 \mathrm{~cm}, \leq 5$ & & $200(50.76)$ & & $138(57.74)$ & \\
\hline$>5 \mathrm{~cm}$ & & 105 (26.65) & & 62 (25.94) & \\
\hline Chemotherapy & 979 & & 512 & & 0.009 \\
\hline Yes & & 322 (32.89) & & $134(26.17)$ & \\
\hline No & & 657 (67.11) & & 378 (73.83) & \\
\hline Radiation & 979 & & 512 & & $<0.001$ \\
\hline Yes & & 297 (30.34) & & 49 (9.57) & \\
\hline No & & $682(69.66)$ & & $463(90.43)$ & \\
\hline Surgery & 967 & & 504 & & 0.151 \\
\hline Yes & & 735 (76.01) & & 366 (72.62) & \\
\hline No & & 232 (23.99) & & $138(27.38)$ & \\
\hline
\end{tabular}

${ }^{a}$ patients with data available.

NRT-SPRC, SPRC without RT; RT-SPRC, SPRC with RT.

\section{DISCUSSION}

Our SEER-based study was the largest patient cohort in the literature and investigated the relationship between pelvic RT as a treatment for PPC and the risk of developing SPRC. Our data confirmed that patients who received pelvic RT for their PPC were at an increased risk of developing SPRC. Recent studies have reached similar conclusions $(4,16,21)$. In addition, our data showed that while a greater proportion of patients who underwent RT also developed SPRC, the proportion of patients who developed SPC after pelvic RT was similar to that for patients who did not receive $\mathrm{RT}$. This indicated that the increased incidence of SPC was associated only with the rectum, which is also the area most likely to be affected by pelvic RT.

Moreover, among patients who received RT for their PPC, the risk of developing a SPRC was higher for patients who received a combination of EBRT and BRT than for those who received EBRT only. Studies have reported that SPC rates were similar overall for high- or low-dose RT treatment for pelvic cancer (risk ratio $(\mathrm{RR})=0.97,95 \% \mathrm{CI}: 0.89-1.06$ ), as were the rates for sitespecific cancers. There was a significant reduction in colon cancer rates following BRT compared with those after EBRT (22). In our study, we did not compare different doses of RT. However, we confirmed that the EBRT-BRT combination increased the SPRC risk compared with EBRT alone, which may have been due to the higher doses of radiation associated with combination treatment. Our results showed that the RTrelated SPRC risk was associated with the pelvic RT treatment modality, which may also be associated with the radiation dose. This difference resulting from the different RT modalities employed or different radiation doses cannot be ignored. We suggest that patients undergoing the EBRT-BRT combination treatment should be considered as having a higher SPRC risk. 
TABLE 3 | Standardized incidence ratio (SIR) of second primary rectal cancer (SPRC).

\begin{tabular}{|c|c|c|c|c|c|c|c|c|c|}
\hline & \multicolumn{3}{|c|}{ NRT } & \multicolumn{3}{|c|}{ EBRT } & \multicolumn{3}{|c|}{ EBRT-BRT } \\
\hline & Observed & SIR & $95 \% \mathrm{Cl}$ & Observed & SIR & $95 \% \mathrm{Cl}$ & Observed & SIR & $95 \% \mathrm{Cl}$ \\
\hline Total & 979 & $0.85 \#$ & $0.80-0.91$ & 325 & $1.22 \#$ & $1.09-1.36$ & 187 & $1.85 \#$ & $1.60-2.14$ \\
\hline \multicolumn{10}{|c|}{ Age at diagnosis (years) } \\
\hline $20-59$ & 82 & 1.12 & $0.89-1.40$ & 8 & 1.21 & $0.52-2.38$ & 18 & 3.00\# & $1.78-4.74$ \\
\hline $60-74$ & 365 & $0.78 \#$ & $0.7-0.87$ & 97 & 1.23 & $1.00-1.50$ & 70 & $1.77 \#$ & $1.38-2.24$ \\
\hline $75+$ & 532 & $0.88 \#$ & $0.81-0.96$ & 220 & $1.21 \#$ & $1.06-1.38$ & 99 & $1.79 \#$ & $1.45-2.18$ \\
\hline \multicolumn{10}{|l|}{ Gender } \\
\hline Male & 632 & $0.80 \#$ & $0.74-0.87$ & 236 & $1.15 \#$ & $1.01-1.31$ & 91 & $1.63 \#$ & $1.32-2.01$ \\
\hline Female & 347 & 0.97 & $0.87-1.08$ & 89 & $1.43 \#$ & $1.15-1.76$ & 96 & $2.12 \#$ & $1.72-2.59$ \\
\hline \multicolumn{10}{|l|}{ Race } \\
\hline White & 837 & 0.83\# & $0.78-0.89$ & 267 & $1.24 \#$ & $1.10-1.40$ & 152 & $1.87 \#$ & $1.59-2.20$ \\
\hline Black & 74 & 0.96 & $0.75-1.21$ & 31 & 1.02 & $0.69-1.44$ & 19 & 1.49 & $0.90-2.33$ \\
\hline Other & 68 & 1.23 & $0.96-1.56$ & 27 & 1.29 & $0.85-1.87$ & 16 & $2.35 \#$ & $1.34-3.82$ \\
\hline \multicolumn{10}{|l|}{ Latency } \\
\hline 60-119 months & 509 & $0.88 \#$ & $0.8-0.96$ & 163 & 1.04 & $0.88-1.21$ & 63 & 1.22 & $0.94-1.56$ \\
\hline 120-239 months & 385 & $0.84 \#$ & $0.76-0.92$ & 135 & $1.39 \#$ & $1.16-1.64$ & 95 & 2.33\# & $1.89-2.85$ \\
\hline $240+$ months & 85 & 0.81 & $0.65-1.00$ & 27 & $2.14 \#$ & $1.41-3.12$ & 29 & $3.42 \#$ & $2.29-4.91$ \\
\hline \multicolumn{10}{|l|}{ Year } \\
\hline 1975-1984 & 18 & 0.85 & $0.51-1.35$ & 2 & 0.51 & $0.06-1.84$ & 1 & 0.50 & $0.01-2.80$ \\
\hline 1985-1994 & 132 & 0.92 & $0.77-1.09$ & 28 & 1.20 & $0.80-1.74$ & 21 & $1.68 \#$ & $1.04-2.57$ \\
\hline 1995-2004 & 271 & 0.92 & $0.81-1.03$ & 62 & 1.13 & $0.87-1.45$ & 35 & $1.67 \#$ & $1.16-2.32$ \\
\hline $2005+$ & 558 & $0.82 \#$ & $0.75-0.89$ & 233 & $1.26 \#$ & $1.10-1.43$ & 130 & $1.99 \#$ & $1.66-2.36$ \\
\hline \multicolumn{10}{|l|}{ Site } \\
\hline Cervix & 49 & 1.22 & $0.90-1.62$ & 19 & 2.94\# & $1.77-4.60$ & 49 & $2.44 \#$ & $1.80-3.22$ \\
\hline Corpus uteri & 211 & 1.00 & $0.87-1.14$ & 65 & 1.27 & $0.98-1.62$ & 47 & $1.87 \#$ & $1.38-2.49$ \\
\hline Ovary & 42 & 1.21 & $0.87-1.64$ & 2 & 0.89 & $0.11-3.22$ & 0 & 0 & $0-100.45$ \\
\hline Prostate & 359 & $0.75 \#$ & $0.67-0.83$ & 220 & 1.13 & $0.99-1.29$ & 91 & $1.63 \#$ & $1.32-2.01$ \\
\hline Bladder & 318 & $0.84 \#$ & $0.75-0.94$ & 19 & 1.45 & $0.87-2.26$ & 0 & 0 & $0-174.55$ \\
\hline
\end{tabular}

SIR, standardized incidence ratios; $C$, confidence interval; NRT, no radiation therapy; EBRT, external beam radiation therapy; EBRT-BRT, combination of external beam with brachytherapy.

${ }^{\#} p<0.05$.

A

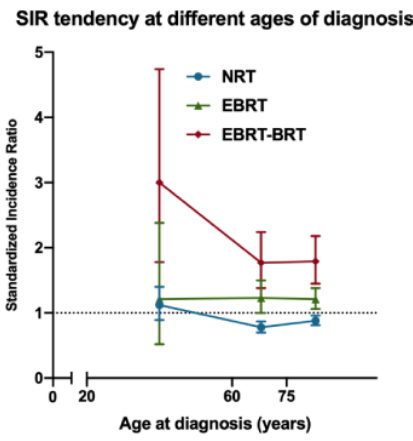

B

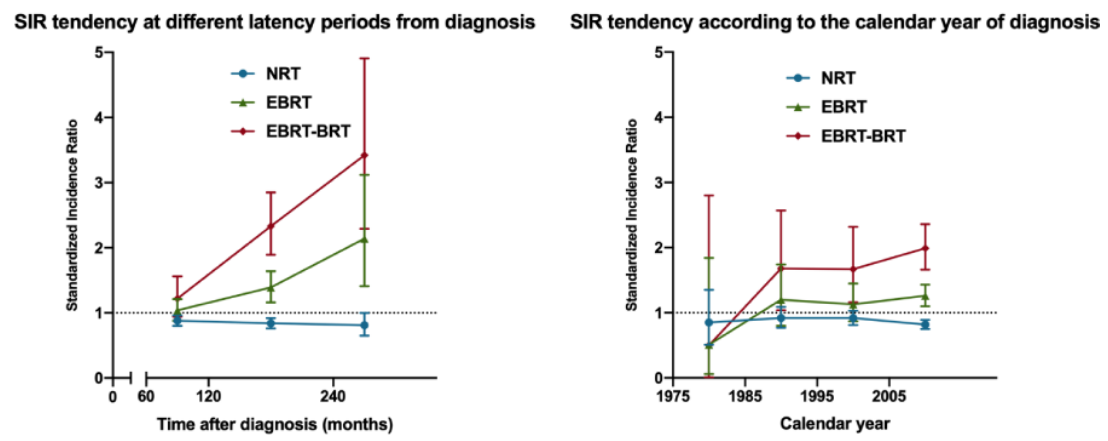

FIGURE 1 | The standardized incidence ratio (SIR) tendency for second primary rectal cancer (SPRC) in surviving pelvic cancer patients. (A) SIR tendency at different ages of diagnosis. (B) SIR tendency at different latency periods from diagnosis. (C) SIR tendency according to the calendar year of diagnosis.

The risk of SPRC appeared to be reduced among patients who did not receive pelvic RT after a PPC, and a similar observation was made for prostate cancer and bladder cancer. These results were consistent with those of other studies $(12,16)$.

We also observed that patients diagnosed with a PPC at a younger age were at the highest risk of developing a SPRC. A study based on a Swedish population showed that the SIRs for colorectal cancer were higher for men diagnosed with Hodgkin lymphoma before the age of 35 years than for those diagnosed later in life (23). Clearly, younger patients would have a longer lifespan after being cured, which also implies a greater cancer risk. We also concluded that the risk of developing SPRC increases with time after a 5 year latency period from PPC diagnosis. This tendency was not observed in the NRT group, indicating that the increased risk of developing SPRC was indeed due to the pelvic RT and not other factors. Considering the 
A

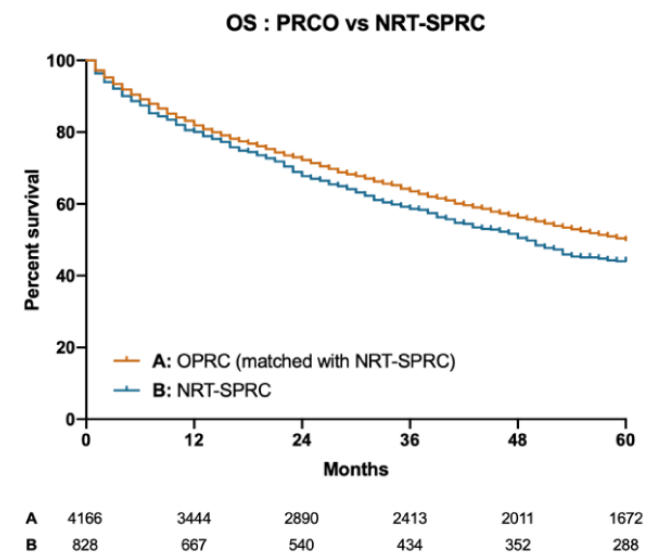

C

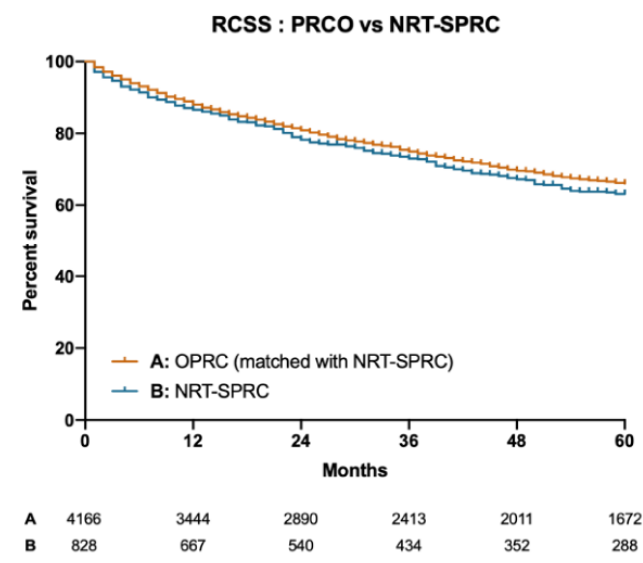

B

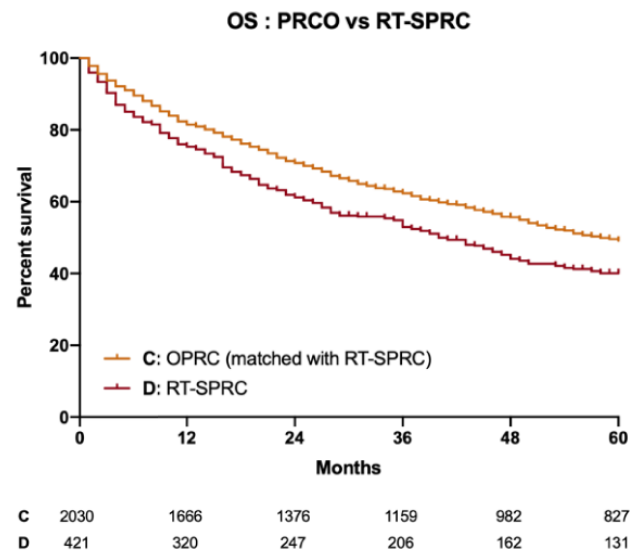

D

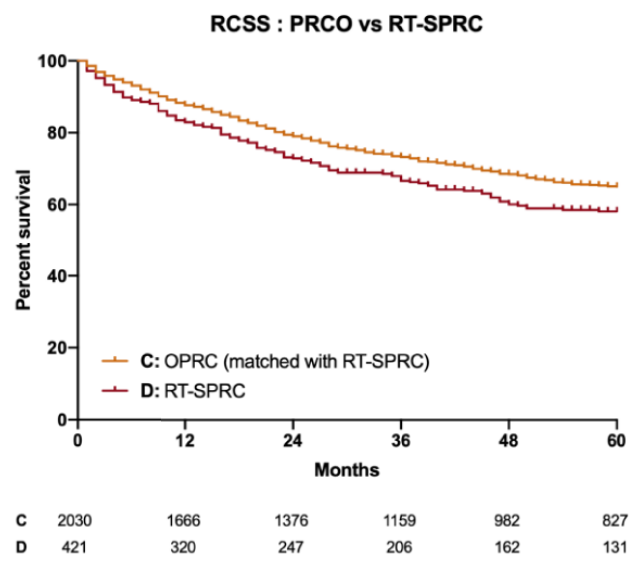

FIGURE 2 | Kaplan-Meier curves for the overall survival (OS) and rectal cancer-specific survival (RCSS) of primary rectal cancer only (PRCO) patients versus those with second primary rectal cancer (SPRC; with radiotherapy [RT] or without radiotherapy [NRT]). (A) OS of PRCO versus NRT-SPRC patients. (B) OS of PRCO versus RT-SPRC patients. (C) RCSS of PRCO versus NRT-SPRC patients. (D) RCSS of PRCO versus RT-SPRC patients.

expanding effect, the latency period of SPRC could be very long indeed. This suggests that a long follow-up is needed for patients undergoing pelvic RT, especially for young patients. Interestingly, we found that the SPRC risk increased with increasing calendar year of diagnosis in RT-treated patients. This tendency did not exist in patients who did not receive RT, indicating that this effect was also due to the RT. With the advancement of RT technology, more cancer patients would have been cured, and an increasing number of pelvic cancer survivors would also result in an increased SPRC potential.

The OS and RCSS were worse for patients in the RT-SPRC group than for those in the PRCO group. For the NRT patients, no significant difference in RCSS was found between them and those in the PRCO group. This indicated that pelvic RT may affect the pathogenesis and biological characteristics of rectal cancer, and lead to differences in survival outcome. Combined with the observation that patients in the RT-SPRC group had a greater incidence of mucinous adenocarcinoma, we suggest that clinicians should consider these differences when treating RT-related SPRC patients and pay special attention to the treatment modality.

This study had several limitations. First, environmental factors that may have had a significant influence on cancer incidence, such as smoking, were not considered as no records were available in the SEER database. Because of the lack of precise RT information in the database, the time frame included in the study does not account for the change to favoring IMRT in many pelvic malignancies. The purpose of this study was to analyze the impact of pelvic RT on SPRC; however, no specific radiation doses were available as a reference, which made it difficult to analyze whether this risk was related to the radiation dose. Nonetheless, as a remedy, we distinguished between patients who underwent EBRT as the only form of RT and those who received a combination of EBRT and BRT. In theory, the EBRT-BRT group should have received higher radiation doses, allowing us to analyze the effect of different RT 
modalities and doses on the risk of developing SPRC. Also, this study is greatly limited by the number of confounders that might be minimized in a Metanalysis from prospective clinical trials.

In conclusion, compared with the U.S. general population, patients who received RT for PPC were at an increased risk of developing a SPRC. Moreover, this RT-related risk for SPRC was associated with the RT treatment modality they had received. We suggest that a long follow-up time is needed for patients undergoing pelvic RT, especially for young patients. Special consideration should be given to SPRC patients given the differences between this group and PRCO patients.

\section{DATA AVAILABILITY STATEMENT}

The raw data supporting the conclusions of this article will be made available by the authors, without undue reservation.

\section{ETHICS STATEMENT}

The studies involving human participants were reviewed and approved by Ethics Committee of National Cancer Center/

\section{REFERENCES}

1. Tabaja L, Sidani SM. Management of Radiation Proctitis. Dig Dis Sci (2018) 63:2180-8. doi: 10.1007/s10620-018-5163-8

2. van Gijn W, Marijnen CAM, Nagtegaal ID, Kranenbarg EM-K, Putter H, Wiggers T, et al. Preoperative radiotherapy combined with total mesorectal excision for resectable rectal cancer: 12-year follow-up of the multicentre, randomised controlled TME trial. Lancet Oncol (2011) 12:575-82. doi: 10.1016/S1470-2045(11)70097-3

3. Baskar R, Dai J, Wenlong N, Yeo R, Yeoh KW. Biological response of cancer cells to radiation treatment. Front Mol Biosci (2014) 1:24. doi: 10.3389/ fmolb.2014.00024

4. de Gonzalez AB, Curtis RE, Kry SF, Gilbert E, Lamart S, Berg CD, et al. Proportion of Second Cancers Attributable to Radiotherapy Treatment in Adults: A Cohort Study in the US SEER Cancer Registries. Lancet Oncol (2011) 12:353-60. doi: 10.1016/S1470-2045(11)70061-4

5. Donin N, Filson C, Drakaki A, Tan HJ, Castillo A, Kwan L, et al. Risk of second primary malignancies among cancer survivors in the United States, 1992 through 2008. Cancer (2016) 122:3075-86. doi: 10.1002/ cncr.30164

6. Lönn S, Gilbert ES, Ron E, Smith SA, Stovall M, Curtis RE. Comparison of second cancer risks from brachytherapy and external beam therapy after uterine corpus cancer. Cancer Epidemiol Biomarkers Prev (2010) 19:464-74. doi: 10.1158/1055-9965.EPI-09-0892

7. Abdel-Wahab M, Reis IM, Hamilton K. Second primary cancer after radiotherapy for prostate cancer-a seer analysis of brachytherapy versus external beam radiotherapy. Int J Radiat Oncol Biol Phys (2008) 72:58-68. doi: 10.1016/j.ijrobp.2007.12.043

8. Birgisson H, Påhlman L, Gunnarsson U, Glimelius B. Occurrence of second cancers in patients treated with radiotherapy for rectal cancer. J Clin Oncol (2005) 23:6126-31. doi: 10.1200/JCO.2005.02.543

9. Huo D, Hetzel JT, Roy H, Rubin DT. Association of colorectal cancer and prostate cancer and impact of radiation therapy. Cancer Epidemiol Biomarkers Prev (2009) 18:1979-85. doi: 10.1158/1055-9965.EPI-09-0241

10. Baxter NN, Tepper JE, Durham SB, Rothenberger DA, Virnig BA. Increased risk of rectal cancer after prostate radiation: a population-based study. Gastroenterology (2005) 128:819-24. doi: 10.1053/j.gastro.2004.12.038
National Clinical Research Center for Cancer/Cancer Hospital. Written informed consent for participation was not required for this study in accordance with the national legislation and the institutional requirements.

\section{AUTHOR CONTRIBUTIONS}

XW: writing-review and editing and supervision. XG: conceptualization, investigation, and supervision. RY: conceptualization, investigation, and writing-original draft. EL: software and formal analysis. RW: validation and data curation. ZZ: resources. HC: validation. ZL: investigation and project administration. MY: data curation and supervision. ZJ: data curation and revision. All authors contributed to the article and approved the submitted version.

\section{SUPPLEMENTARY MATERIAL}

The Supplementary Material for this article can be found online at: https://www.frontiersin.org/articles/10.3389/fonc.2020. 584072/full\#supplementary-material

11. Majewski W, Majewski S, Maciejewski A, Kolosza Z, Tarnawski R. Adverse Effects After Radiotherapy for Early Stage (I,IIa,IIb) Seminoma. Radiother Oncol (2005) 76:257-63. doi: 10.1016/j.radonc.2005.04.003

12. Rombouts AJM, Hugen N, Elferink MAG, Poortmans PMP, Nagtegaal ID, de Wilt JHW. Increased Risk for Second Primary Rectal Cancer After Pelvic Radiation Therapy. Eur J Cancer (2020) 124:142-51. doi: 10.1016/j.ejca.2019. 10.022

13. Hinnen KA, Schaapveld M, van Vulpen M, Battermann JJ, van der Poel H, van Oort IM, et al. Prostate Brachytherapy and Second Primary Cancer Risk: A Competitive Risk Analysis. J Clin Oncol (2011) 29:4510-5. doi: 10.1200/ JCO.2011.35.0991

14. Wiltink LM, Nout RA, Fiocco M, Kranenbarg EM-K, Jürgenliemk-Schulz IM, Jobsen JJ, et al. No Increased Risk of Second Cancer After Radiotherapy in Patients Treated for Rectal or Endometrial Cancer in the Randomized TME, PORTEC-1, and PORTEC-2 Trials. J Clin Oncol (2015) 33:1640-6. doi: 10.1200/JCO.2014.58.6693

15. Murray LJ, Thompson CM, Lilley J, Cosgrove V, Franks K, Sebag-Montefiore $D$, et al. Radiation-induced Second Primary Cancer Risks From Modern External Beam Radiotherapy for Early Prostate Cancer: Impact of Stereotactic Ablative Radiotherapy (SABR), Volumetric Modulated Arc Therapy (VMAT) and Flattening Filter Free (FFF) Radiotherapy. Phys Med Biol (2015) 60:123757. doi: 10.1088/0031-9155/60/3/1237

16. Rombouts AJM, Hugen N, van Beek JJP, Poortmans PMP, de Wilt JHW, Nagtegaal ID. Does Pelvic Radiation Increase Rectal Cancer Incidence? - A Systematic Review and Meta-Analysis. Cancer Treat Rev (2018) 68:136-44. doi: 10.1016/j.ctrv.2018.05.008

17. Werbrouck J, Ost P, Fonteyne V, De Meerleer G, De Neve W, Bogaert E, et al. Early biomarkers related to secondary primary cancer risk in radiotherapy treated prostate cancer patients: IMRT versus IMAT. Radiother Oncol (2013) 107:377-81. doi: 10.1016/j.radonc.2013.05.014

18. van der Meij W, Rombouts AJM, Rütten H, Bremers AJA, de Wilt JHW. Treatment of Locally Recurrent Rectal Carcinoma in Previously (Chemo) Irradiated Patients: A Review. Dis Colon Rectum (2016) 59:148-56. doi: 10.1097/DCR.0000000000000547

19. Valentini V, Morganti AG, Gambacorta MA, Mohiuddin M, Doglietto GB, Coco C, et al. Preoperative Hyperfractionated Chemoradiation for Locally Recurrent Rectal Cancer in Patients Previously Irradiated to the Pelvis: A 
Multicentric Phase II Study. Int J Radiat Oncol Biol Phys (2006) 64:1129-39. doi: 10.1016/j.ijrobp.2005.09.017

20. Margel D, Baniel J, Wasserberg N, Bar-Chana M, Yossepowitch O. Radiation therapy for prostate cancer increases the risk of subsequent rectal cancer. Ann Surg (2011) 254:947-50. doi: 10.1097/SLA.0b013e3182382fd5

21. Preston DL, Ron E, Tokuoka S, Funamoto S, Nishi N, Soda M, et al. Solid cancer incidence in atomic bomb survivors: 1958-1998. Radiat Res (2007) 168:1-64. doi: 10.1667/RR0763.1

22. de Gonzalez AB, Wong J, Kleinerman R, Kim C, Morton L, Bekelman JE. Risk of Second Cancers According to Radiation Therapy Technique and Modality in Prostate Cancer Survivors. Int J Radiat Oncol Biol Phys (2015) 91:295-302. doi: 10.1016/j.ijrobp.2014.10.040

23. Sud A, Thomsen H, Sundquist K, Houlston RS, Hemminki K. Risk of Second Cancer in Hodgkin Lymphoma Survivors and Influence of
Family History. J Clin Oncol (2017) 35:1584-90. doi: 10.1200/JCO.20 16.70.9709

Conflict of Interest: The authors declare that the research was conducted in the absence of any commercial or financial relationships that could be construed as a potential conflict of interest.

Copyright (C) 2020 Yang, Guan, Liu, Wei, Zhao, Chen, Liu, Yang, Jiang and Wang. This is an open-access article distributed under the terms of the Creative Commons Attribution License (CC BY). The use, distribution or reproduction in other forums is permitted, provided the original author(s) and the copyright owner(s) are credited and that the original publication in this journal is cited, in accordance with accepted academic practice. No use, distribution or reproduction is permitted which does not comply with these terms. 